\title{
AD ASTRA PER ASPERA
}

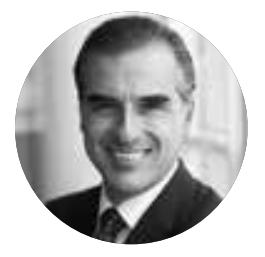

\author{
Andrés A. Escalante \\ ORCID: 0000-0002-6937-5578 \\ Editor General \\ Profesor de Economía Empresarial \\ Facultad de Negocios, UPC \\ Lima, Perú. \\ andres.escalante@upc.pe
}

Cómo citar este artículo:

Escalante, A. (2020). Ad Astra Per Aspera.

Review of Global Management, 6(1), 04-06. as oficinas de esta revista, Review of Global Manage-

ment, están en el Área Académica de Administración

de la UPC. Esta área está adscrita organizacionalmente

a la Facultad de Negocios y su gobierno es la responsabilidad de la Dirección Académica de Administración y Finanzas (DAF), uno de los siete brazos ejecutivos de dicha facultad. La Facultad de Negocios tiene una cultura organizacional, consistente con una misión, que, lógicamente, se subsume en la de la UPC. Si bien quienes formamos parte de la Facultad de Negocios trabajamos en términos de su cultura, la relativa autonomía de cada una de sus direcciones académicas le permite a su personal profesar valores adicionales, que, si bien tienen que ser consistentes con dicha cultura, no tienen por quéser los mismos. Es así como en la DAF tenemos un decálogo de valores que rige todas y cada una de nuestras decisiones laborales e inclusive extra-laborales. ${ }^{1}$ Pero lo interesante, en este caso, no es tanto el decálogo en sí, como el lema que circunda a estos valores cual conjunto: Ad astra per aspera. Este lema, escrito en latín, puede traducirse como, "a las estrellas a través de las dificultades".

No encuentro mejor expresión para describir la resiliencia que necesitamos tener individual, como colectivamente, tal es el santo y seña de nuestro tiempo, para enfrentar la coyuntura actual y que, se hace patente, ha de convertirse en la tormenta perfecta social y económicamente. Enfrentar los avatares funestos de esta pandemia, entre la inminente crisis sanitaria y las consecuencias económicas de un confinamiento social que, indefectiblemente, pondrán en tela de juicio a las medidas asumidas por la Administración del presidente Vizcarra, con miras a una crisis política

${ }^{1}$ Este decálogo de valores está disponible a pedido. posible, significa cumplir, en la medida de lo viable, con nuestros compromisos laborales, sorteando óbices; y es así, como, después de un gran "trabajo de parto", ve la luz este nuevo y esperado número de nuestra revista: et lux in tenebris lucet. ${ }^{2}$

Este número, que su merced está por disfrutar, se compone, además de esta nota editorial, de cuatro artículos a lo largo de casi 40 páginas, escritos por investigadores de reputadas instituciones académicas de formación superior locales. Entre sus páginas, asimismo, se contempla una interesantísima entrevista, sin medias tintas, a Mariana Rodríguez, una de los fundadores de la UPC, con motivo del vigesimoquinto aniversario de vida institucional de esta casa de estudios. Estos ensayos, como la entrevista, se condicen con nuestra pretensión de generar una mayor lectoría por su multiplicidad temática. Presentan reflexiones limpias, puntos de vista formales, alturada discusión y análisis rigurosos sobre temas que, dada nuestra actual realidad, resultan oportunos, estructural, como coyunturalmente: libertad económica; gestión del riesgo y la cadena de suministro; el impacto de la pandemia en ciernes en la formación de habilidades blandas en particular y la educación superior en general, como en el desempeño laboral y el valor de la educación superior.

En el primer ensayo, Beltrán Gómez (Instituto Político para la Libertad), nos presenta las ideas centrales de la filosofía de la libertad, en la que el individuo es el protagonista indiscutible y cuya libertad debe protegerse frente a la coacción de terceros, particularmente del Estado, mientras

${ }^{2}$ Frase en latín que significa: y la luz brilló en medio de la oscuridad. 
nos relata reflexivamente su conversión, durante su vida universitaria, en un defensor de la libertad, conforme descubría la obra intelectual de algunas de las mentes más brillantes de dicha filosofía. De este relato, nos queda clara la vigencia actual de las principales ideas sobre el comportamiento humano y las instituciones que lo norman en sociedad, de gigantes como, Carl Menger, Ludwig von Mises y Friedrich von Hayek, entre otros.

En el segundo ensayo, Oscar Zamudio (UPC) y Alex Izquierdo (UPC), reconocen que las complejidades de la cadena suministro imponen grandes retos organizacionalmente, por lo que es imperioso una gestión afín resiliente. Este punto, del que parten como premisa, no es menor, toda vez que los riesgos de gestión en la cadena de suministro surgen inesperada y hasta agresivamente y no siempre la organización está lista para asumirlos adecuadamente. Es por ello que, en su investigación, sustentando la importancia de usar métodos que eviten o neutralicen las posibles disrupciones en diversas situaciones, apelan a la gestión integral de riesgos, como una herramienta medular para tamaño propósito.

En el tercer artículo, Lida Vásquez-Pajuelo (UPC), Doris Vila-Gómez (Universidad San Ignacio de Loyola) y Jazmín Tuesta-Vila (Universidad Peruana del Norte), investigan cómo desarrollar en el estudiante universitario las habilidades blandas en el marco de la pandemia en ciernes. Ello, con el fin de empoderarlos con mayor autonomía, para identificar sus propias cualidades socioemocionales, cementando la base para tener un desarrollo emocional armónico profesionalmente. Arguyen, por ello, primeramente, entender qué son las habilidades blandas; y, en segundo lugar, que las universidades fomenten las distintas alternativas de formación temática respectiva. Esta investigación, cualitativa en su naturaleza, pasa revista de la literatura disponible sobre el tema, como de la data, para abordar la inquietud de encontrar la forma de permitir que los estudiantes desarrollen estas habilidades, intuitiva, como dinámicamente, mas no sin la asistencia docente.En el cuarto y último ensayo, Victoria Chuco (Universidad Nacional Mayor de San Marcos), José Álvarez ((Universidad Nacional Mayor de San Marcos), Manuel Chávez ((Universidad Nacional Mayor de San Marcos) y Luis Cuba (Universidad Nacional Mayor de San Marcos), analizan la relación entre el teletrabajo y la productividad laboral en las organizaciones. El teletrabajo se estaría convirtiendo en el principal medio para laborar en las empresas, pero, ineluctablemente, esto representa un reto para ellas en cuanto a productividad laboral se refiere, en parte por la falta de infraestructura física, como virtual, con el agravante de la presión laboral y la incertidumbre en el mercado de trabajo por la tesitura circundante. Del estudio, se desprende la importancia de establecer una debida comunicación entre el tele-trabajador y el resto de la organización, y de la capacidad del empleado para administrar sus emociones ante la incertidumbre.

El esfuerzo por divulgar conocimiento, por parte de la UPC, es constante. La Review of Global Management, representa uno de los medios que la UPC tiene para tamaño cometido. En ella, se abordan temas principalmente relacionados con la administración de empresas y los negocios globales, la economía y los mercados, las finanzas, la innovación y el emprendimiento, entre otros. La revista tiene por objeto proveer al lector de la inteligencia necesaria para tres funciones generales. Primero, la de emprender negocios propios; corporativizar negocios familiares; y fomentar el intra-emprendimiento en las organizaciones establecidas y formales. En segundo lugar, la función de resaltar la rentabilidad de la ética en las organizaciones y el valor de la buena gobernanza. Tercero, la de fomentar institucionalmente la innovación.

Si bien la temática es amplia, en la revista se apuesta, principalmente, por un enfoque analítico del problema bajo estudio. Este, por definición, es subsumible en un razonamiento lógico en el que la teoría ejerce la rectoría organizacional. Ello permite formular hipótesis, invocar principios, aplicar conceptos y emplear herramientas metodológicamente. Se trata, pues, de abordar este problema con elegancia académica, para lograr, en términos de resultados robustos, posiciones defendibles, y conclusiones serias, por ejemplo, formular recomendaciones útiles o redefinir el estado del arte, como los límites del conocimiento.

La revista, como no puede ser de otra manera, se ajusta a los estándares institucionales y normas internacionales de publicación y registro, en cuanto a formato. Lo que publica es material inédito y se hace en consistencia con el derecho de propiedad intelectual. Asimismo, la calidad de los aportes es materia de cuidado, por la que se vela celosamente, y por tanto, se someten a la evaluación oficial por parte del Comité Editorial Internacional de la revista.

En el marco del liderazgo consciente, mediante la Review of Global Management, tenemos como parte de nuestro propósito ulterior institucional, estrechar lazos con 


\section{REVIEW OF GLOBAL}

\section{MANAGEMENT}

FACULTAD

DE NEGOCIOS

DE LA UPC

nuestros grupos de interés, como ratificar la presencia de esta casa de estudios en la sociedad peruana en su capacidad generadora de conocimiento. Esta revista es una iniciativa que, en su sexto año, continúa proyectándose, gracias a la expectativa que genera en nuestros lectores su publicación periódica. Sin embargo, no por ello hemos de desconocer que esta crisis sanitaria internacional nos puede "trazar la cancha" de otra forma, haciendo que la manera como trabajamos actualmente resulte inadecuada para emprender eficaz y eficientemente. De ser así, resultaría matemático tener que reagruparnos para repensar las cosas y dotarnos de nuevas herramientas para abordar los problemas desde alguna otra arista, porque, como diríamos en inglés, it might be time to "regroup, rethink, and retool."3 Es la única forma de llegar a las estrellas a través de las dificultades.

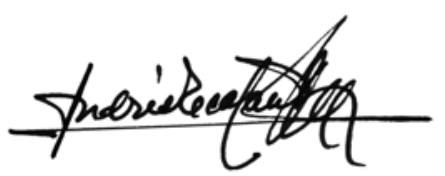

Andrés A. Escalante, Ph.D. EDITOR GENERAL

\section{REFERENCIAS}

Chuco, V., Álvarez, J., Chávez, M., \& Cuba, L. (2020). El trabajo remoto y el desempeño laboral en el marco del COVID-19. Review of Global Management, 6(1), 50-55.

Escalante, A. (2020). Entrevista a Mariana Rodríguez Risco. Review of Global Management, 6(1), 35-40.

Gómez, B. (2020). Los pensadores de la libertad-o un universo escondido entre papeles sueltos-. Review of Global Management, 6(1), 07-13.

Vásquez-Pajuelo, L., Vila-Gómez, D. A., \& Tuesta-Vila, J. A. (2020). Habilidades blandas y el impacto de la covid-19 en la educación superior. Review of Global Management, 6(1), 41-49.

Zamudio, O., \& Izquierdo, A. (2020). Modelo de Gestión de Riesgo de la Cadena de Suministro como Elemento Diferenciador. Review of Global Management, 6(1), 14-34. 